\section{Neues ESC-Positionspapier: Tumorpatient braucht auch den Kardiologen}

\begin{abstract}
Antitumoröse Therapien sind mit einer Reihe von kardialen Risiken assoziiert. Deshalb erfordert der Tumorpatient auch kardiologischen Sachverstand. Die Europäische Gesellschaft für Kardiologie hat jetzt ein entsprechendes Positionspapier herausgebracht.
\end{abstract}

$\mathrm{D}$ as Thema „Herz und Krebs“ wird angesichts der demografischen Entwicklung, aber auch der Entwicklung neuer Therapiestrategien immer wichtiger", darauf machte Prof. Stephan Achenbach, Direktor der kardiologischen Universitätsklinik in Erlangen/ Nürnberg aufmerksam. Dem wolle man mit einem Positionspapier Rechnung

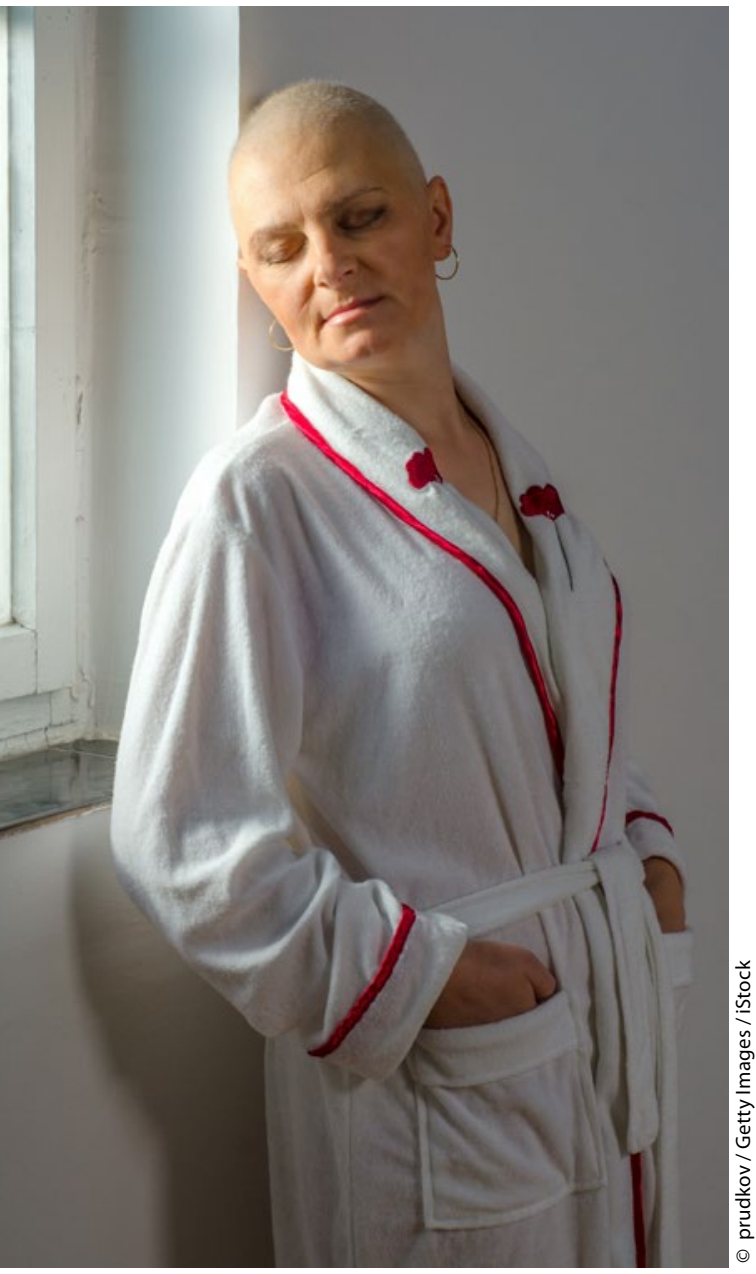

Bei Brustkrebspatientinnen kann eine Chemotherapie mit Anthracycline schon früh eine toxische Kardiomyopathie verursachen. tragen, welches jetzt auf der ESC-Jahrestagung in Rom vorgestellt wurde. Es wurde gemeinsam von der ESC und der ICOS (International CardioOncology Society) erarbeitet und umfasst alle Aspekte dieses Themenkomplexes.

\section{Toxische Kardiomyopathie durch Anthracycline}

Die bekannteste kardiale Komplikation im Rahmen der Tumortherapie ist die durch Anthracycline verursachte toxische Kardiomyopathie bei Frauen mit Mammakarzinom. Diese limitiert den Einsatz dieser Substanz vor allem bei älteren Patientinnen. Insgesamt dürften in Abhängigkeit von der Dosis und dem Alter 10 bis $45 \%$ der damit behandelten Frauen betroffen sein. Sie kann sehr früh nach der ersten Gabe oder nach einigen Monaten und gar nicht so selten erst viele Jahre nach der Chemotherapie auftreten.

So haben Kinder, die mit einem Anthracyclin mit oder ohne Strahlentherapie des Mediastinums behandelt wurden, ein 15-fach erhöhtes Risiko während ihres Lebens eine Herzinsuffizienz zu entwickeln im Vergleich zu Personen ohne Tumorleiden.

Risikofaktoren für die toxische Kardiomyopathie sind neben der kumulativen Dosis weibliches Geschlecht, chronische Niereninsuffizienz, vorbestehende kardiale Erkrankungen bzw. arterielle Hypertonie und eine begleitende kardiotoxische Medikation. Deshalb sollte vor Einleitung der Chemotherapie der Blutdruck optimal eingestellt sein.

\section{Regelmäßige Echokardiografie- Kontrollen}

Um die Manifestation der toxischen Kardiomyopathie frühzeitig erkennen zu können, sind regelmäßige Echokardiografie-Kontrollen erforderlich. Das entscheidende diagnostische Kriterium sind eine Abnahme der Ejektionsfraktion $(\mathrm{EF})>10 \%$ vom Ausgangswert.

Eine beginnende myokardiale Schädigung lässt sich mit den herkömmlichen Echoparametern wie der EF jedoch nicht zuverlässig erfassen. Dazu bedarf es zusätzlicher Techniken wie dem SpeckleTracking-Imaging oder dem Gewebedoppler. Für eine myokardiale Schädigung spricht eine Veränderung des globalen longitudinalen Strain um $>15 \%$.

Im Rahmen einer beim ESC vorgestellten Studie wurde der Frage nachgegangen, ob Nebivolol kardioprotektiv wirkt, also die Manifestation einer Anthracyclin-Kardiomyopathie verhindern kann. Eingeschlossen wurden 60 Frauen mit Mammakarzinom, die randomisiert mit $5 \mathrm{mg}$ Nebivolol einmal täglich behandelt wurden. „Durch den Betablocker konnte die Toxizität günstig beeinflusst werden“, berichtete Prof. Mirela Tomescu von der Universität in Timişoara/Rumänien. Unter Nebivolol ergaben sich in der Echokardiografie keinerlei Hinweise für eine myokardiale Schädigung im Unterschied zu Patienten ohne diese Begleittherapie.

\section{Weitere Risiken zu beachten}

Aber nicht nur die Herzinsuffizienz, sondern auch andere kardiale therapiebedingte Risiken müssen bei einer antitumorösen Behandlung bedacht werden. Dazu gehören die KHK und die degenerativen Veränderungen an den Herzklappen vor allem die Aortenstenose, und zwar als langfristige Folge einer thorakalen Strahlentherapie.

"Gerade die Interaktionen zwischen Tumortherapie und koronarer Herzerkrankung, also die kardiovaskuläre Toxizität sind bisher noch kaum erforscht, obwohl sie durchaus relevant sind", beonte Achenbach. Mögliche Mechanismen seien Vasospasmen, die endotheliale Dysfunktion und eine prothrombotische Begleitwirkung der eingesetzten Substanzen.

Dr. med. Peter Stiefelhagen

2016 ESC Position Paper on cancer treatments and cardiovascular toxicity developed under the auspices of the ESC Committee for Practice GuidelinesThe Task Force for cancer treatments and cardiovascular toxicity of the European Society of Cardiology (ESC), Eur Heart J 2016, online 27. August, DOI: 10.1093/eurheartj/ehw272 